\title{
Adaptive Immune Responses Mediated Age-related Plasmodium yoelii 17XL and 17XNL Infections in 4 and 8-week-old BALB/c Mice
}

\section{Qiu-bo Wang}

China Medical University

\section{Yun-ting Du}

Cancer hospital of China Medical University

Fei Liu

College basic sciences, China Medical University

Xiao-dan Sun

College basic sciences, China Medical University

Xun Sun

College basic sciences, China Medical University

\section{Guang Chen}

Taizhou University

\section{Wei Pang}

China Medical University

ya-ming Cao (Dymccmu@163.com )

China Medical University

\section{Research article}

Keywords: age-related, Plasmodium yoelii 17XL, Plasmodium yoelii 17XNL, adaptive immune responses, 4-week-old BALB/c mice, 8-week-old BALB/c mice

Posted Date: October 7th, 2019

DOl: https://doi.org/10.21203/rs.2.15639/v1

License: (c) (i) This work is licensed under a Creative Commons Attribution 4.0 International License. Read Full License

Version of Record: A version of this preprint was published at BMC Immunology on January 11th, 2021. See the published version at https://doi.org/10.1186/s12865-020-00391-8. 


\section{Abstract}

Background As the quest to eradicate malaria continues, it is important to clarify the opposite clinical outcomes between childhood and adulthood. The relationship between adaptive immune response and age-related malaria infection remains unknown.

Methods 4 and 8-week-old mice were used to mimic childhood and adulthood, respectively. Parasitemia and the survival rate were monitored. The proportion and function of Th1 and Th2 cells were detected by FACS. The levels of IFN-ץ, IL-4, total IgG, IgG1, IgG2a and Plasmodium yoelii MSP-1-special IgG were measured by ELISA.

Results Infant mice were more susceptible to P. yoelii 17XNL infection, with lower survival rate and higher parasitemia. The adult group showed greater resistance to P. yoelii 17XL infection, with lower parasitemia. Compared with 4-week-old mice, the percentage of CD4 + T-bet + IFN- + + Th1 cells as well as IFN- $y$ production were significantly increased on day 5 p.i. in the 8-week-old mice after P. yoelii $17 \mathrm{XNL}$ infection. The percentage of CD 4 + GATA3 + IL-4 + Th2 cells and CD 4 + CXCR5 + Tfh cells, and IL-4 production in the 8-week-old mice obviously increased on day 5 and day 10 after P. yoelii 17XNL infection. Notably, the levels of total IgG, IgG1, IgG2a and P. yoelii MSP-1-special IgG were also significantly increased in the 8-week-old mice. PD-1, a marker of exhaustion, was up-regulated on CD4 + or activated CD4 + T cells in the 8-week-old mice as compared to the 4-week-old group.

Conclusion We consider that enhanced cellular and humoral adaptive immunity might contribute to rapid clearance of malaria among adults, likely in a PD-1-dependent manner due to induction of CD $4+\mathrm{T}$ cells exhaustion.

\section{Background}

Malaria is a major cause of mortality and morbidity in tropical countries [1]. According to the recent WHO statistics, 91 countries reported ongoing malaria transmission, with Africa experiencing disproportionately high malaria cases ( $90 \%$ of the total) and accounting for $91 \%$ of total malaria deaths worldwide [2]. Notably, children under the age of 5 years are particularly vulnerable to plasmodium infection. More than two-thirds of malaria deaths (70\%) occur in this age group [3]. Of the five Plasmodium species that infect humans, Plasmodium falciparum and Plasmodium vivax are the most common, and $P$. falciparum is the most virulent and responsible for the majority of deaths $[2,3]$. In addition, the multiplicity of infection (MOI) varies depending on the overall prevalence of infection in the population, and the age of the individual $[4,5]$. The young children are highly susceptible to clinical illness and high parasitemia, whereas the adults are highly resistant [4], resulting in a major difference in the spectrum of disease manifestations between children and adults [6]. Therefore, understanding the immunological mechanisms involved in susceptibility to different virulent Plasmodium species during infection in childhood or adulthood could contribute to the development of an immunologically based control strategy to prevent or treat this devastating disease. 
Upon infection, anti-parasite immunity plays a central role in removing the parasite from the blood. Innate immunity is first activated, e.g., complement system, innate lymphoid cells and dendritic cells (DCs) act to limit the acute phase of parasitemia, but are insufficient to clear the infection $[7,8,9]$. When DCs present the processed antigen, adaptive immunity is activated. Direct cell cytotoxicity, cytokine secretion as well as anti-malarial antibody work together for effective parasite clearance $[10,11,12,13]$.

Infants and young children are more susceptible to malaria infection than adults worldwide [4]. Agerelated changes in immune systems increased prevalence of asthma, nasal polyps and lung injury [14, 15]. However, whether differences in cellular and humoral immunity lead to this age-related infection profile remains unknown. Therefore, we used different virulent Plasmodium (lethal $P$. $y 17 X L$ and nonlethal $P$. $y 17 X N L$ ) strains to infect4-week-old and 8-week-old BALB/c mice to mimic infancy and adulthood, respectively, in order to characterize the relationship between immune cell responses and agerelated malaria infection among different age groups, and understand the mechanism of malaria immunity. We propose that the dynamics of $\mathrm{MOI}$ can be explained by a model of increasing acquired immunity to blood-stage infection with age.

\section{Methods}

\section{Mice, Parasite and experimental infection}

The 4-week-old and 8-week-old female BALB/c mice were purchased from Beijing Animal Institute. $P$. $y 17 \mathrm{XL}$ and $P . y 17 \mathrm{XNL}$ strains were provided by Dr. Motomi Torii (Department of Molecular Parasitology, Ehime University Graduate School of Medicine, Ehime, Japan). Infections were initiated by intraperitoneal (i.p.) injection of $1 \times 10^{6} P$. $y 17 \mathrm{XL}$ or $1 \times 10^{6}$ P. y $17 \mathrm{XNL}$ parasitized erythrocytes in BALB/c mice. The experimental mice were matched for age and sex. Parasitemia was examined by light microscopy of Giemsa-stained, tail blood smears. Mortality was monitored daily. All experiments were performed in compliance with local animal ethics committee requirements. The animals were not submitted to euthanasia during the process of plasmodium infection, which all mice died in day 7-11 p.i.in P.y 17XLinfected mice.

\section{Spleen cell culture}

Spleen cell culture was prepared as previously described [16]. Briefly, spleen was aseptically removed from each mouse and passed through a sterile fine-wire mesh with $10 \mathrm{ml}$ of RPMI1640 (Life Technologies, Burlington, Ontario, Canada) supplemented with $5 \%$ heat-inactivated fetal calf serum (FCS) (Hyclone Laboratories, Inc.), 25mM Hepes (Life Technologies), 0.12\% gentamicin (Schering, Montreal, Quebec, Canada) and 2mM glutamine (Life Technologies). Cell suspensions were centrifuged at 350×g for $10 \mathrm{~min}$ at room temperature (RT). Erythrocytes were lysed with cold $0.17 \mathrm{M} \mathrm{NH}_{4} \mathrm{Cl}$. The cells were washed twice with fresh medium. Viability of the spleen cells was determined by trypan blue exclusion, and was always $>90 \%$. Spleen cells were adjusted to a final concentration of $10^{7}$ cells $/ \mathrm{ml}$ in RPMI 1640 
supplemented with $10 \%$ heat-inactivated FCS. Aliquots $(500 \mu \mathrm{l} /$ well) of the cell suspension were incubated in 24-well flat-bottom culture plates (FALCON) in triplicate for 48 hours at $37^{\circ} \mathrm{C}$ in a humidified $5 \% \mathrm{CO}_{2}$ incubator. Then, the plates were centrifuged at $350 \times \mathrm{g}$ for $10 \mathrm{~min}$ at RT, supernatants were collected and stored at $-80^{\circ} \mathrm{C}$ until they were assayed for the levels of IFN-g, IL-4, IgG, IgG1, IgG2a and $P$. y MSP-1-special IgG.

\section{Cytokine analysis}

Levels of IFN-g and IL-4 were measured by commercial enzyme-linked immunosorbent assay (ELISA) kits according to the manufacturer's protocols (R\&D Systems, Minneapolis, MN). The OD values were read in a microplate reader at $450 \mathrm{~nm}$. The concentrations of cytokines in samples were calculated against the standard curve generated using recombinant IFN- $y$ and IL -4 , respectively.

\section{Multiplex assay for antibody determination}

Levels of total serum IgG, IgG1, IgG2a and $P$. $y$ MSP-1-special IgG were measured by ELISA as previously described with some modifications [17]. Briefly, Maxisorp flat-bottomed, 96-well microplates were coated overnight at $4^{\circ} \mathrm{C}$ with $50 \mu \mathrm{g}$ of $P . y \mathrm{MSP}-1$ antigens in a carbonate-bicarbonate buffer (pH 9.6). The plates were washed with PBS-Tween (PBS-T) and blocked with $0.05 \%$ bovine serum albumin (BSA)-PBST. Next, $100 \mu \mathrm{l}$ of plasma dilutions in $0.05 \%$ BSA-PBS-T (1:50 for $P$. $y$ MSP-1 lgG) were added in duplicate and incubated at RT for $2 \mathrm{~h}$. After washing with PBS-T, the plates were incubated with horseradish peroxidase-conjugated goat anti-mouse IgG (Sigma, USA) at a dilution of 1:5000. The OD values were read in a microplate reader at $490 \mathrm{~nm}$.

\section{Cell surface/intracytoplasmicstaining and flow cytometry}

To assess the function of $\mathrm{CD} 4^{+} \mathrm{T}$ cells, spleen cells from BALB/c mice infected with $P$. $y 17 X L / P . y 17 X N L$ at different time points were double-stained with FITC-conjugated anti-CD4 (clone GK1.5, BD), BV421conjugated anti-PD-1 (clone J43, BD), PE-conjugated anti-CXCR-5 (clone 2G8, BD) and APC-conjugated anti-CD62L (MEL-14, BD), followed by two washes, staining and analysis by flow cytometry.

To assess dynamics of Th1 and Th2 cells, spleen cells from BALB/c mice infected with $P$. $y 17 X L / P$. y17XNL at different time points were triple-stained with fluorescein isothiocyanate (FITC)-conjugated antiCD4 (clone GK1.5), PE-conjugated anti-T-bet (clone eBio4B10, eBioscience), APC-conjugated anti-IFN- $\gamma$ (XMG1.2, BD) for Th1 cells, and FITC-conjugated anti-CD4 (clone GK1.5), PE-conjugated anti-GATA-3 (clone L50-823, BD), APC-conjugated anti-IL-4 (clone 11B11, BD) for Th2 cells. After stimulation for 2 hours with PMA and ionomycin at $37^{\circ} \mathrm{C}$, Golgi Stop (BD Bioscience) was added to each reaction (1:500, $\mathrm{vol} / \mathrm{vol}$ ). After co-culture for 4 hours at $37^{\circ} \mathrm{C}$, the cells were washed with $3 \% \mathrm{FCS}$ and then resuspended in $100 \mu \mathrm{l}$ of $3 \% \mathrm{FCS}$. FITC-anti-CD4, PE-anti-T-bet and PE-anti-GATA3were added for surface staining. Then, the cells were fixed and permeabilized, and intracytoplasmic staining was performed using 
allophycocyanin (APC)-anti-IFN-Y/IL-10 (JES5-16E3). FITC rat IgG2b was used as the isotype control. All antibodies were purchased from BD Pharmingen.

\section{Statistical analysis}

All analyses were performed using GraphPad Prism version 6.0 (GraphPad Software, La Jolla, CA). Data are presented as mean \pm standard error of the mean (SEM). Survival analysis was performed using the Kaplan-Meier log-rank test. Statistical significance of differences between the two groups was assessed by unpaired Student's t-tests. P-values were calibrated using Bonferroni correction, and were considered statistically significant if they were less than 0.05 .

\section{Results}

\section{Comparison of different species of Plasmodium infection course in 4- week-old and 8-week-old BALB/c mice}

To investigate the relationship between age-related host immunity against malaria infection, we used $\mathrm{BALB} / \mathrm{c}$ mice of different age groups to mimic infancy and adulthood, and monitored parasitemia and the survival rate at different time points after lethal $P . y 17 X L$ and non-lethal $P . y 17 X N L$ infections. Within 20 days after $P$. $y 17 X N L$ infection, $96 \%$ of the 8-week-old mice successfully survived whereas only $78 \%$ of the 4-week-old mice survived (Fig. 1A). In accordance with the survival rate, the parasitemia peaked at $12 \%$ in the 4-week-old mice on day 11 p.i. while it was only $7 \%$ in the 8-week-old group, although the onset of parasitemia was similar in both groups on day 3 p.i. (Fig. 1B). Similarly, parasitemia peaked at $80 \%$ in the 4-week-old mice on day 8 p.i., and all mice died; however, in the 8-week-old group, parasitemia peaked at $75 \%$ on day 8 p.i., subsequently declined, and all mice died on day 11 p.i. (Fig. 1D). Although both groups were sensitive to lethal P. $y 17 X L$ infection and failed to control parasite growth, the 8-week-old mice seemed to be more resistant to lethal infection with slower death rate (Fig. 1C) and lower parasitemia during the early stage of malaria infection (Fig. 1D). Therefore, infants were more susceptible to parasite infection, whereas the adult group seemed to be relatively resistant.

Comparison of Th1 immune response in different species of Plasmodium-infected 4-week-old and 8week-old $B A L B /$ c mice

Next, the relationship between Th1 cell responses and age during the early stage of malaria infection was determined. The percentage of $\mathrm{CD} 4^{+} \mathrm{T}^{-}$bet ${ }^{+} \mathrm{IFN}-\mathrm{\gamma}^{+} \mathrm{Th} 1$ cells was determined by flow cytometry, and the level of IFN- $\gamma$ in splenocytes was measured by ELISA. Compared with 4-week-old mice, the frequency and absolute number of Th1 cells were significantly increased in P. y17XNL-infected 8-week-old mice on day 5 p.i. (Fig. 2A) $(\mathrm{p}<0.05)$. The level of IFN-y in $P$. $y 17 X N L$-infected 8-week-old mice on day 5 p.i. had the same trend as Th1 cells (Fig. 2C). Interestingly, in $P$. $y 17 X L$ infected 8-week-old mice, the frequency and absolute number of Th1 cells peaked on day 3 p.i. (Fig. $2 B)(p<0.05)$, then subsequently decreased, but remained higher than normal control on day 5 p.i. $(\mathrm{p}<0.05)$. Notably, the level of IFN-y in lethal $P . y 17 X L-$ 
infected mice was significantly increased on day 5 p.i. $(p<0.05)$, but there was no obvious difference between the 4-week-old mice and 8-week-old mice. This data suggested that enhanced Th1 cell responses might be associated with age-related non-lethal $P$. y17XNL infection and resistance during the early stage of lethal $P$. $y 17 X L$ infection.

Comparison of Th2 immune response in different species of Plasmodium-infected 4-week-old and 8week-old $B A L B /$ c mice

To assess the characteristics of Th2 cell responses and their relationship with age during the late stage of malaria infection, we evaluated the percentage and absolute number of $\mathrm{CD} 4^{+} \mathrm{GATA} 3^{+} \mathrm{IL}-4^{+} \mathrm{Th} 2$ cells and interleukin-4 (IL-4) production. The proportion and absolute number of Th2 cells were elevated in both groups on day 5 p.i. and day 10 p.i. after P. y17XNL infection as compared to normal control (Fig. $3 A, B)(p<0.05)$. However, there was no obvious difference in the percentage of Th2 cells between the 4week-old and 8 week-old BALB/c mice. Consistently, the level of IL-4 production in P. y17XNL-infected 8week-old mice was significantly increased as compared to the 4-week-old mice on day 5 p.i. and day 10 p.i. (Fig. 3B) (p<0.05). In addition, we detected the percentage and absolute number of $\mathrm{CD} 4^{+} \mathrm{CXCR} 5^{+} \mathrm{Tfh}$ cells, recognized as specialized providers of cognate B cell help. The percentage and absolute number of $\mathrm{CD} 4^{+} \mathrm{CXCR} 5^{+}$Tfh cells peaked on day 5 p.i., and then decreased to normal level on day 10 p.i. in the 4week-old mice. However, in the 8-week-old mice, the percentage and absolute number of $\mathrm{CD} 4^{+} \mathrm{CXCR} 5^{+} \mathrm{Tfh}$ cells were significantly increased on day 10 p.i. as compared to the 4-week-old mice (Fig. 3D) $(p<0.05)$. As expected, the percentage and absolute number of Th2 cells, and $\mathrm{CD} 4^{+} \mathrm{CXCR} 5^{+} \mathrm{Tfh}$ cells, and the level of IL-4 were significantly elevated in $P$. y $17 X N L$ infected 8-week-old group during the late stage of malaria infection. These results indicated that an enhanced Th2 immunity during non-lethal $P$. $y 17 X N L$ infection might contribute to rapid clearance of Plasmodium in adults.

\section{PD-1 expression promotes immune response in different species of Plasmodium-infected 4-week-old and 8-week-old BALB/c mice}

PD-1 signaling plays an essential role in regulating immune cell exhaustion. To explore whether PD-1 signaling mediated effector $T$ cell exhaustion and facilitated persistent infection in infancy or adulthood, we detected the expression of PD-1 on $\mathrm{CD}^{+}$or activated $\mathrm{CD} 4^{+} \mathrm{T}$ cells after lethal P. $y 17 X L$ and nonlethal $P$. $y 17 X N L$ infection by flow cytometry. The expression of PD-1 on $\mathrm{CD} 4^{+}$or activated $\mathrm{CD} 4^{+} \mathrm{T}$ cells was obviously increased on day 5 and day 10 p.i. after lethal P. y17XL and non-lethal $P$. $y 17 X N L$ infections. Compared with 8-week-old mice, the expression of PD-1 on $\mathrm{CD} 4^{+}$or activated $\mathrm{CD} 4^{+} \mathrm{T}$ cells after non-lethal $P$. $y 17 X N L$ infection on day 10 p.i. was significantly raised in the 4-week-old mice (Fig. 4A, B) (p<0.05). Interestingly, in lethal P. $y 17 X L$ infection, the expression of PD -1 on $C D 4^{+}$or activated $C D 4^{+} T$ cells was obviously higher in the 8-week-old mice than in the 4-week-old mice on day 10 p.i. (Fig. 4C, D). 


\section{The levels of total and P. y MSP-1-specific antibody in P. y17XNL- infected 4-week-old and 8-week-old BALB/c mice}

Protection from clinical malaria has been reported to be associated with both the breadth and magnitude of the antibody responses to merozoite antigens [18]. ELISA of B cell-related total IgG, IgG1 and IgG2a also showed a significant difference in antibody production in adult mice as compared to infant mice (Fig. 5A, B, C). Interestingly, compared with 4-week-old mice, P. y MSP-1-specific IgG antibody production was obviously increased in the 8-week-old mice during malaria infection (Fig. 5D) $(p<0.05)$.

\section{Discussion}

Malaria infection is known to be age-related, with children being more susceptible than adults $[19,20,21$, 22]. This study aimed to investigate whether the susceptibility to malaria infection in childhood and adulthood is associated with cellular and humoral immune responses, using a mouse model of lethal $P$. $y 17 X L$ and non-lethal $P . y 17 X N L$ infections in different age groups. Infant mice were found to be more susceptible to $P . y 17 X N L$ infection, with lower survival rate and higher parasitemia at various time points. The adult group was more resistant to $P . y 17 X L$ infection with lower parasitemia during the early stage of malaria infection. Importantly, enhanced cellular and humoral immunity, especially MSP-1 special antibody, might contribute to rapid clearance of malaria in the adult group.

Malaria infections have various clinical phenotypes, ranging from a mild febrile illness to life-threatening severe anemia and acidosis, as well as end-organ failure among individuals with little or no acquired antimalarial immunity. In part, this is explained by heritable differences in susceptibility to malaria infections, including different parasite proliferation rates governed by erythrocyte and hemoglobin polymorphisms [23].Both the strain and host were thought to be important determinants of the disease profile [24]. In this study, 4-week-old and 8-week-old mice were used to mimic infancy and adulthood, respectively. We successfully established the age-related malaria infection mouse model to study the age-related antimalaria immunity. The survival rate and parasitemia at different time points indicated that the 4-week-old group was more susceptible to both lethal and non-lethal parasite infections. After non-lethal $P$. y17XNL infection, parasitemia was significantly higher in the 4-week-old mice than the 8-week-old mice during the acute and chronic stages of infection. After lethal $P . y 17 X L$ infection, a significant difference in parasitemia was observed in the early stage of infection. In accordance with the parasitemia, enhanced Th1 immune responses were only observed in the early stage (day 3 ) in adult mice after lethal $P . y 17 X L$ infection and enhanced adaptive immune responses were detected in adult mice during both the early and late stages of non-lethal P. $y 17 X N L$ infection. These data suggested that the difference in response to non-lethal and lethal Plasmodium infections was associated with the pattern of immune cell responses in the host.

Similar to other infectious diseases, accumulating evidences have indicated that CD $4^{+} \mathrm{T}$ cells are essential to control malaria infection $[25,26,27,28]$. Numerous studies have highlighted the role of Th1/Th2 cells or related signaling mechanisms in controlling malaria infection [29, 30, 31, 32, 33]. In this 
study, enhanced Th1 and Th2 responses were displayed in 8-week-old mice after malaria infection. Significantly higher percentage of Th1/Th2 cells and level of IFN- $/$ IL-4 were observed in the 8-week-old mice as compared to the 4-week-old mice. In vitro studies also showed an enhanced Th1 cell response, which indicated an important role of Th1/Th2 cell-mediated age-related anti-malarial response. However, many studies suggested a shift from Th2 to Th1 cell responses with age. Li et al. found that IFN- $\gamma$ level increased with age but not Th-related transcription factors, while IL-4 expression in plasma and CD $4^{+}$ splenocytes declined with age [34]. A shift from Th2 towards Th1 immune responses was also observed in children with tertian or tropical malaria infection [35]. These studies partly supported our conclusion that enhanced Th1 cells might contribute to malaria clearance during the early stage of malaria infection. However, we observed enhanced Th2 cells during the late stage/chronic stage of malaria infection. Further studies are needed to investigate if any shift exists during the early stage of malaria infection. In addition, follicular T helper (Tfh) cells are essential for Plasmodium infection clearance by activating germinal center $B$ cell responses $[36,37,38,39]$. In this study, the percentage of $C D 4^{+} C X C R 5^{+}$Tfh cells was also up-regulated in adult mice as compared to the infant group. Moreover, Crompton PD et al. found that the activation of $\mathrm{PD}-1^{+} \mathrm{CXCR} 5^{+}$Tfh cells in malaria-infected children did not correlate with antibody responses indicating that Tfh cells may exhibit impaired B cells in children, which is different from Tfh cells in adults [40]. These studies supported our findings that the impaired function of antibody-secreting $B$ cells and Tfh cells in infants and children may account for their susceptibility to malaria infection.

We also observed a dampening of PD-1 signaling on activated CD $4^{+} \mathrm{T}$ cells after non-lethal $P$. $y 17 X N L$ infection but not lethal $P . y 17 X L$ infection in the 8-week-old mice. $P D-1$ co-inhibitory signaling was reported to regulate helper $T$ cell differentiation and anti-Plasmodium humoral immunity [41], and PD-1 deficiency could enhance humoral immunity during malaria infection [42]. PD-1 was also a marker of Tcell exhaustion [43]. Several studies have also proven that chronic malaria infection drives T cell exhaustion through PD-1 signaling $[44,45]$. Therefore, we speculated that during non-lethal infection, humoral immunity plays an essential role in the late stage of malaria clearance, perhaps correlated with enhanced PD-1 signaling on activated $C D 4^{+} T$ cells, which may help to drive $C D 4^{+}$effector $T$ cell exhaustion and promote persistent infection in infants. Therefore, differences in PD-1 signaling could be observed in different age groups after non-lethal but not lethal malaria infection.

Several studies have confirmed that immune effector mechanisms are required to eliminate malarial parasites, and B cells secrete specific antibodies supported by Th2 cells, which can effectively remove the parasites to prevent the recidivation and recrudescence $[46,47]$. Similarly, infusion of malaria hyperimmune serum resulted in rapid clearance of parasitized erythrocytes [47]. Anti-Plasmodium antibodies can prevent merozoites of infected red blood cells (RBCs), block cytoadherence to endotheliar capillary of infected RBCs (iRBCs), and promote phagocytosis by mononuclear cells $[48,49,50]$. However, persistence of significant levels of antimalarial antibodies relies on the continuous challenge resulting from chronic exposure to infection [51], probably as a consequence of impaired establishment of B cell memory [52]. This might explain the short-lived antibody responses [53,54], mainly in young children [55, 56]. In this study, we detected the levels of B cell-related total IgG, IgG1 and IgG2a in P. y 17XNL-infected 
BALB/c mice. The results showed a difference in antibody production between adult and infant mice, and the levels of total antibody might contribute to rapid clearance of malarial parasites in the adult group during the chronic stage of non-lethal P. y17XNL infection. Moreover, IgG1 and IgG3 antibodies against merozoite surface proteins (MSPs) are thought to be instrumental in protection, which is considered as a major vaccine candidate [57]. Therefore, we detected the levels of $P$. $y \mathrm{MSP}-1$ special antibody. Consistently, the dynamics of $P . y \mathrm{MSP}-1$ special antibody was the same as total antibody. These data implied that an enhanced antibody response during chronic stage of non-lethal $P$. $y 17 X N L$ infection might contribute to rapid clearance of malaria in the adult group.

\section{Conclusions}

Taken together, the findings of this study revealed that in non-lethal $P$. $y 17 X N L$ infection, higher burden of parasitemia and lower survival rate in infant mice were associated with weakened Th1 cellular immune responses, down-regulated humoral immunity with decreased percentage and number of Th2 and Tfh cells as well as lower level of antibody secretion and enhanced PD-1 signaling on activated $\mathrm{CD} 4^{+} \mathrm{T}$ cells. Higher resistance to lethal $P . y 17 X L$ infection in the early stage in adult mice was associated with enhanced Th1 cellular immune responses and weakened PD-1 signaling on activated CD ${ }^{+} \mathrm{T}$ cells. These results provide a new insight on immune responses in malaria infection.

\section{Abbreviations}

P.y17XL:Plasmodium yoelii 17XL; P.y17XNL: Plasmodium yoelii 17XNL; i.p.: intraperitoneal; PBS: phosphate-buffered saline; FCS: fetal calf serum; RT: room temperature; ELISA: enzyme linked immunosorbent assay.

\section{Declarations}

- Acknowlegements

We thank Dr. Motomi Torii (Ehime University Graduate School of Medicine, Ehime, Japan) for his guidance in this research and providing malaria parasite strains of P.y17XL.

- Ethics approval and consent to participate

Ethical approval was obtained from the Ethical Review Committee (ERC) at China Medical University.

- Consent for publication

Not applicable.

- Availability of data and materials

Please contact the author for data requests. 
- Competing interests

The authors declare that they have no conflicts of interest in relation to this article.

- Funding

This study was supported in part by funds from the National Natural Science Foundation of China (81101278) 『key project of the natural science foundation in Heilongjiang Province (ZD2017020)『doctor star-up foundation of Liaoning Province (20180540019).

- Authors' contributions

Y. M.,C, G. C. and W. P. designed and developed the research study; Q. B.W, Y. T.D, F.L, X. D.S and X. S. performed experiments, analyzed and discussed data, G. C. wrote the paper. All authors read and approved the final manuscript.

- Acknowledgment

We thank Dr. Motomi Torii (Ehime University Graduate School of Medicine, Ehime, Japan) for his guidance in this research and providing malaria parasite strains of P.y17XL and P.y17XNL.

\section{Authors' detail}

${ }^{1}$ Department of Immunology, College of Basic Medical Sciences, China Medical University,No.77 Puhe Rood,Shenyang North New Area, Shenyang, 110122, China; ${ }^{2}$ Department of Clinical Laboratory, Wuxi 9th Affiliated Hospital of Soochow University, No. 999 Liang Xi Road, Binhu District, Wuxi, 214000,China;

${ }^{3}$ Department of Laboratory Medicine, Cancer Hospital of China Medical University, Liaoning Cancer Hospital \& Institute, NO. 44 Xiaoheyan Road, Dadong District, Shenyang 110042, China; ${ }^{4}$ Department of basic medical sciences, Taizhou University medical school, No 1139 shifu road, jiaojiang district, Taizhou 317700, china

\section{References}

1. Beri D, Balan B, Tatu U. Commit, hide and escape: the story of Plasmodium gametocytes.Parasitolog, 2018; 16:1-11. 2. WHO World Malaria Report, 2017. https://www.worldaware.com/article/blog/globalmalaria -report-2017. 3. Miller LH, Baruch DI, Marsh K, et al. The pathogenic basis of malaria. Nature, 2002; 415: 673-679. 4. Pinkevych M, Petravic J, Bereczky S, et al. Understanding the relationship between Plasmodium falciparum growth rate and multiplicity of infection. Journal of Infectious Diseases,2015; 211(7):1121-1127. 5. Soulama I, Nébié I, Ouédraogo A, et al. Plasmodium falciparum genotypes diversity in symptomatic malaria of children living in an urban and a rural setting in Burkina Faso. Malaria Journal, 2009; 8:135. 6. Wassmer SC, Taylor TE, Rathod PK, et al. Investigating the Pathogenesis of Severe Malaria: A Multidisciplinary and Cross-Geographical Approach. American Journal of Tropical Medicine 
and Hygiene.2015; 93(3 Suppl):42-56. 7. Boyle MJ, Reiling L, Feng G, et al. Human antibodies fix complement to inhibit Plasmodium falciparum invasion of erythrocytes and are associated with protection against malaria. Immunity, 2015; 42:580-590. 8. Mauduit M, See P, Peng K, et al. Dendritic cells and the malaria pre-erythrocytic stage. Immunologic research, 2012; 53:115-1126. 9. Palomo J, Quesniaux V, Togbe D, et al. Unravelling the Roles of Innate Lymphoid Cells in Cerebral Malaria Pathogenesis. Parasite Immunology, 2018; 40(2). 10. Berg A, Otterdal K, Patel S, et al. Complement Activation Correlates With Disease Severity and Contributes to Cytokine Responses in Plasmodium falciparum Malaria. The Journal of infectious diseases, 2015; 212:1835-1840. 11. Chen L, He Z, Qin L,et al. Antitumor effect of malaria parasite infection in a murine Lewis lung cancer model through induction of innate and adaptive immunity. PLoS One,2011; 6: e24407. 12. Claser C, Chang ZW, Russell B, et al. Adaptive immunity is essential in preventing recrudescence of Plasmodium yoelii malaria parasites after artesunate treatment. Cellular Microbiology, 2017; 19(11). 13. Mandala WL, Msefula CL, Gondwe EN, et al. Cytokine Profiles in Malawian Children Presenting with Uncomplicated Malaria, Severe Malarial Anemia, and Cerebral Malaria. Clinical and vaccine immunology, 2017; 24(4). pii: e00533-16. 14. Cho SH, Kim DW, Lee $\mathrm{SH}$, et al. Age-related increased prevalence of asthma and nasal polyps in chronic rhinosinusitis and its association with altered IL- 6 trans-signaling. American journal of respiratory cell and molecular biology, 2015; 53:601-606. 15. Linge HM, Lee JY, Ochani K, et al. Age influences inflammatory responses, hemodynamics, and cardiac proteasome activation during acute lung injury. Experimental lung research, 2015; 41: 216-227. 16. Ma SH, Zheng L, Liu YJ, et al. Plasmodium yoelii: influence of antimalarial treatment on acquisition of immunity in BALB/c and DBA/2 mice. Experimental Parasitology, 2007; 116(3):266-272. 17. Mehrizi, A.A., Asgharpour, S., Salmanian, A.H., et al. IgG subclass antibodies to three variants of Plasmodium falciparum merozoite surface protein-1 (PfMSP-1(19)) in an area with unstable malaria transmission in Iran. Acta Tropica, 2011; 119, 84-90. 18. Osier FH, Fegan G, Polley SD, et al. Breadth and magnitude of antibody responses to multiple Plasmodium falciparum merozoite antigens are associated with protection from clinical malaria. Infection and Immunity, 2008; 76: 2240-2248. 19. Boutlis CS, Weinberg JB, Baker $\mathrm{J}$, et al. Nitric oxide production and nitric oxide synthase activity in malaria-exposed Papua New Guinean children and adults show longitudinal stability and no association with parasitemia.Infection and Immunity, 2004; 72(12)『6932-6938. 20. Cox, M. J., D. E. Kum, L. Tavul, A. et al. Dynamics of malaria parasitaemia associated with febrile illness in children from a rural area of Madang, Papua New Guinea. Transactions of the Royal Society of Tropical Medicine and Hygiene. 1994; 88:191-197. 21. Rogier, C., D. Commenges, and J. F. Trape. Evidence for an age-dependent pyrogenic threshold of Plasmodium falciparum parasitemia in highly endemic populations. American Journal of Tropical Medicine and Hygiene, 1996; 54:613-619. 22. Smith, T., B. Genton, K. Baea, et al. Relationships between Plasmodium falciparum infection and morbidity in a highly endemic area. Parasitology, 1994; 109:539-549. 23. Weatherall DJ, Miller LH, Baruch DI, et al. Malaria and the red cell. Hematology (Am Soc Hematol Educ Program). 2002; 1: 35-57. 24. Snounou G, Viriyakosol S, Jarra W, et al. Identification of the four human malaria parasite species in field samples by the polymerase chain reaction and detection of a high prevalence of mixed infections. Molecular and Biochemical Parasitology, 1993; 58:283-292. 25. Couper, K.N., Phillip,s R.S., Brombacher, F., et al. Parasite-specific IgM plays a significant role in the protective immune response to asexual erythrocytic stage Plasmodium chabaudi ASinfection. Parasite 
Immunology, 2005; 27:171-180. 26. Seixas, E., Ostler, D. Plasmodium chabaudi chabaudi (AS): differential cellular responses to infection in resistant and susceptible mice. Experimental Parasitology, 2005; 110:394-405. 27. Taylor-Robinson, A.W., Phillips, R.S. B cells are required for the switch from Th1 to Th2 regulated immune responses to Plasmodium chabaudi infection. Infection and Immunity, 1994; 62:490498. 28. Yazdani, S.S., Mukherjee, P., Chauhan, V.S., et al. Immune responses to asexual blood-stages of malaria parasites. Current Molecular Medicine, 2006; 6:187-203. 29. Fauconnier M, Palomo J, Bourigault $\mathrm{ML}$ et al. IL-12R beta 2 is essential for the development of experimental cerebral malaria. Journal of Immunology, 2012; 188:1905-1914. 30. Haque A, Best SE, Montes de Oca M, James KR et al. Type I IFN signaling in CD8- DCs impairs Th1-dependent malaria immunity. The Journal of clinical investigation, 2014; 124:2483-2496. 31. Maneekan P, Leaungwutiwong P, Misse D, et al. T helper (Th) 1 and Th2 cytokine expression profile in dengue and malaria infection using magnetic bead-based bio-plex assay. The Southeast Asian journal of tropical medicine and public health, 2013; 44:31-36. 32. Perez-Mazliah D, Langhorne J. CD4 T-cell subsets in malaria: TH1/TH2 revisited. Frontiers in Immunology, 2014; $5: 671$. 33. Tatfeng YM, Agbonlahor DE, Amegor OF. Measurement of Th1, Th2 cytokines and white cell count in childhood haemoglobinopathies with uncomplicated malaria infection. Hematology, 2012; 17: 47-50. 34. Xia Y, Yang J, Wang G, et al. Age-Related Changes in DNA Methylation Associated with Shifting Th1/Th2 Balance. Inflammation, 2016; 39:1892-1903. 35. Khodzhaeva NM. Age-related cytokine regulation in children with malaria]. Meditsinskaia parazitologiia i parazitarnye bolezni, 2011;25-28. 36. Figueiredo MM, Costa PAC, Diniz SQ, et al. T follicular helper cells regulate the activation of B lymphocytes and antibody production during Plasmodium vivax infection. PLoS Pathogens, 2017; 13: e1006484. 37. Hansen DS, Obeng-Adjei N, Ly A, et al. Emerging concepts in T follicular helper cell responses to malaria. International Journal for Parasitology, $2017 ; 47: 105-110$. 38. Perez-Mazliah D, Nguyen MP, Hosking C, et al. Follicular Helper T Cells are Essential for the Elimination of Plasmodium Infection. EBioMedicine, 2017; 24:216-230. 39. Salles EM, Menezes MN, Siqueira R, et al. P2X7 receptor drives Th1 cell differentiation and controls the follicular helper $T$ cell population to protect against Plasmodium chabaudi malaria. PLoS Pathogens, 2017; 13: e1006595. 40. Obeng-Adjei N, Portugal S, Tran TM, et al. Circulating Th1-Cell-type Tfh Cells that Exhibit Impaired B Cell Help Are Preferentially Activated during Acute Malaria in Children. Cell reports, 2015; 13: 425-439. 41. Zander RA, Obeng-Adjei N, Guthmiller JJ, et al. PD-1 Co-inhibitory and OX40 Co-stimulatory Crosstalk Regulates Helper T Cell Differentiation and AntiPlasmodium Humoral Immunity. Cell Host $₫$ Microbe. 2015; 13;17(5):628-41. 42. Liu T, Lu X, Zhao C, et al. PD-1 deficiency enhances humoral immunity of malaria infection treatment vaccine. Infection and Immunity, 2015; 83: 2011-2017. 43. Liu J, Zhang S, Hu Y, et al. Targeting PD-1 and Tim-3 Pathways to Reverse CD8 T-Cell Exhaustion and Enhance Ex Vivo T-Cell Responses to Autologous Dendritic/Tumor Vaccines. Journal of immunotherapy, 2016; 39: 171-180. 44. Horne-Debets JM, Faleiro R, Karunarathne DS, et al. PD-1 dependent exhaustion of CD8+ T cells drives chronic malaria. Cell reports, 2013; 5:12041213. 45. Wykes MN, Horne-Debets JM, Leow CY, et al. Malaria drives T cells to exhaustion. Frontiers in microbiology, 2014; .5:249. 46. Maestre A, Carmona-Fonseca J. Immune responses during gestational malaria: a review of the current knowledge and future trend of research.Journal of Infection in Developing Countries, 2014; 8(4):391-402. 47. White NJ. Malaria parasite clearance. Malaria Journal, 2017; 16(1):88 48. Beeson JG, Osier FH, Engwerda CR. 2008. Recent insights into humoral and cellular immune 
responses against malaria. Trends in Parasitology, 24:578-584. 49. Taylor-Robinson AW. Regulation of immunity to Plasmodium: implications from mouse models for blood stage malaria vaccine design. Experimental Parasitology, 2010; 126:406-414. 50. Wipasa J, Elliott S, Xu H, et al. Immunity to asexual blood stage malaria and vaccine approaches. Immunology and Cell Biology, 2002; 80:401-414. 51. Langhorne J, Ndungu FM, Sponaas AM, et al. Immunity to malaria: more questions than answers. Nature Immunology, 2008; 9:725-732. 52. Illingworth J, Butler NS, Roetynck S, et al. Chronic exposure to Plasmodium falciparum is associated with phenotypic evidence of $B$ and $T$ cell exhaustion. Journal of Immunology, 2013; 190:1038-1047. 53. Cavanagh DR, Elhassan IM, Roper C, et al. A longitudinal study of type-specific antibody responses to Plasmodium falciparum merozoite surface protein-1 in an area of unstable malaria in Sudan. Journal of Immunology, 1998; 161:347-359. 54. Dorfman JR, Bejon P, Ndungu FM, et al. B cell memory to 3 Plasmodium falciparum blood-stage antigens in a malaria-endemic area. Journal of Infectious Diseases, 2005; 191:1623-1630. 55. Akpogheneta OJ, Duah NO, Tetteh KK, et al. Duration of naturally acquired antibody responses to blood-stage Plasmodium falciparum is age dependent and antigen specific. Infection and Immunity, 2008;76:1748-1755. 56. Taylor RR, Egan A, McGuinness $D$, et al. Selective recognition of malaria antigens by human serum antibodies is not genetically determined but demonstrates some features of clonal imprinting. International Immunology, 1996; 8:905-915. 57. Walker KM, Okitsu S, Porter DW, et al. Antibody and T-cell responses associated with experimental human malaria infection or vaccination show limited relationships. Immunology. 2015; 145(1):71-81.

\section{Figures}


A

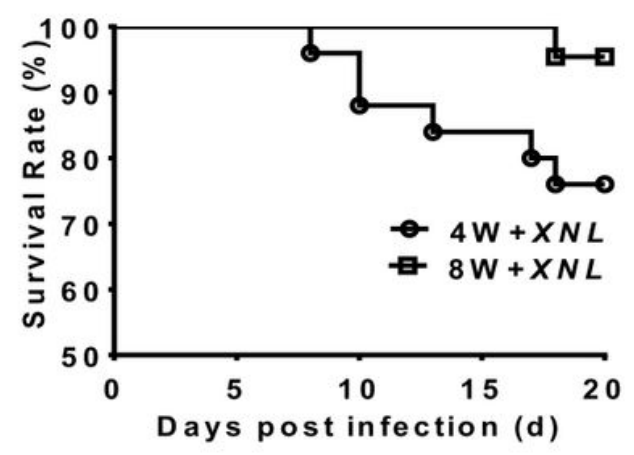

C

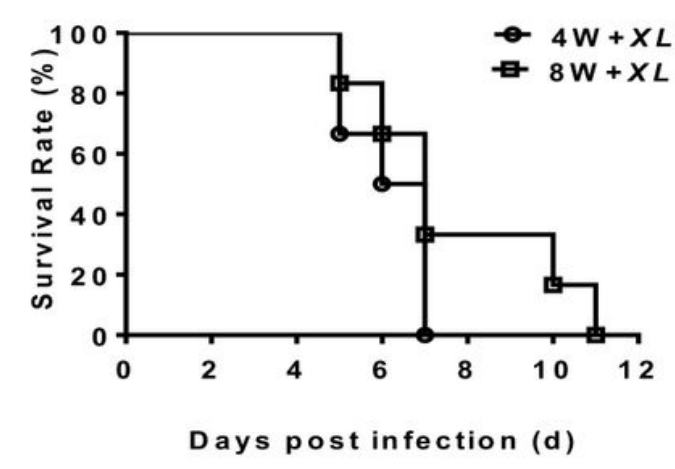

B

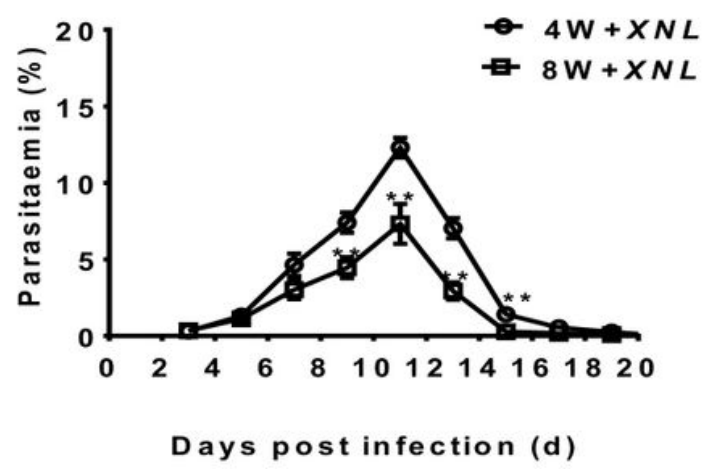

D

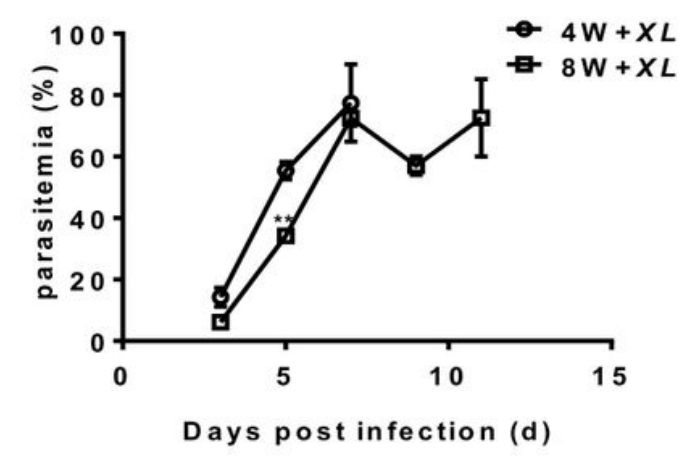

Figure 1

Parasitemia $(A, C)$ and survival rate $(B, D)$ of Py17.XNL or Py17.XL infection in 4-week-old and 8-week-old $\mathrm{BALB} / \mathrm{c}$ mice. Parasitemia was calculated by counting the number of parasite-infected erythrocytes per 1000 erythrocytes. Mortality was checked daily. The data are representative of two separate experiments. 


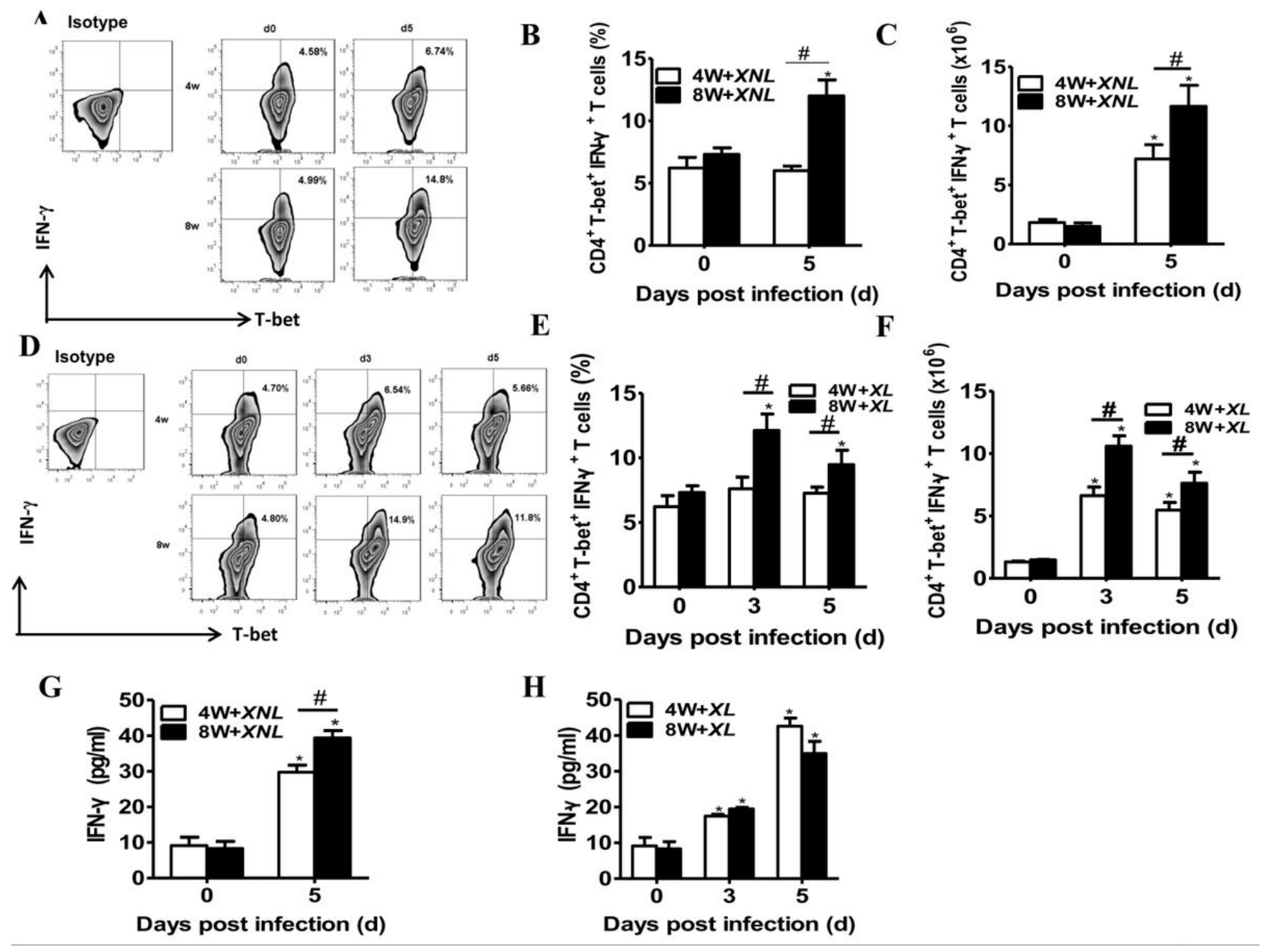

Figure 2

Flow cytometric and ELISA analysis demonstrated Th1 immune response in different species of Plasmodium-infected 4-week-old and 8-week-old BALB/c mice.Two-dimensional contour map (left-upper panel) andcolumn diagram (left-upper panel, the proportion of Th1 cells in CD4+ T cells, absolute cell number) of CD4+T-bet+IFN- $\gamma+$ Th1 cells in 4-week-old and 8-week-old BALB/c mice after Py17XL infection are displayed (A); Representative dot plots (left-lower panel) andcolumn diagram (left-lower panel, the proportion of Th1 cells in CD4+ T cells, absolute cell number) of CD4+T-bet+IFN- $\gamma+$ Th1 cells in 4-weekold and 8-week-old BALB/c mice after Py17XNL infection are displayed (B). Level of IFN-Y (C, D) in spleen cell supernatants in Py17XNL/Py17XL-infected BALB/c mice were measured. Results are presented as arithmetic mean of 3 mice per group \pm SE. Single asterisk $(*)$ and double asterisks $\left({ }^{*}\right)$ indicate $p<0.05$ and $p<0.01$, respectively, as compared to control mice. A single pound sign (\#) and a double pound sign $(\# \#)$ indicate $p<0.05$ and $p<0.01$, respectively, as compared to 8-week-old mice. The data are representative of two separate experiments. 


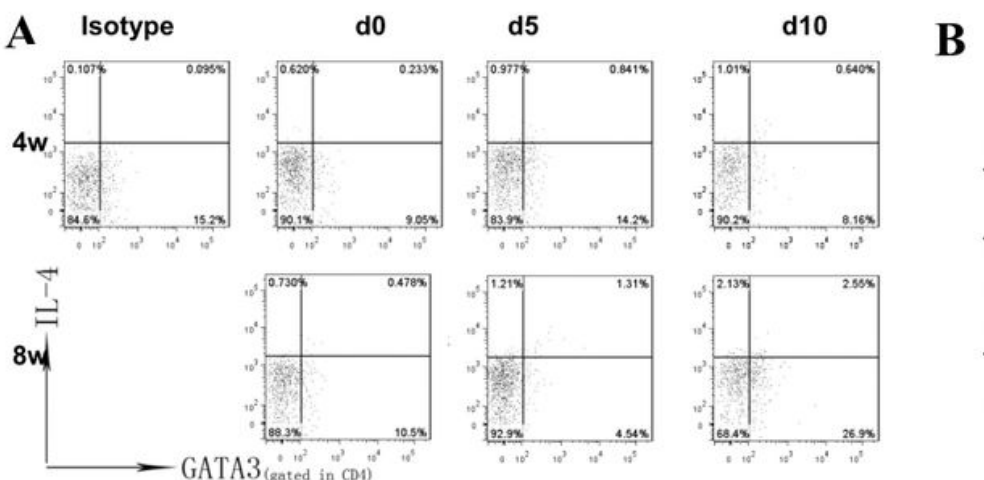

D

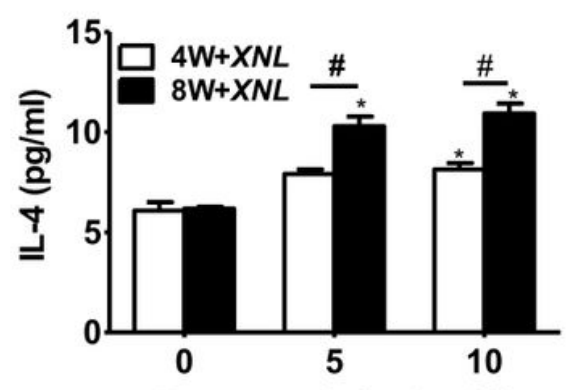

H

Days post infection (d) $X N L$

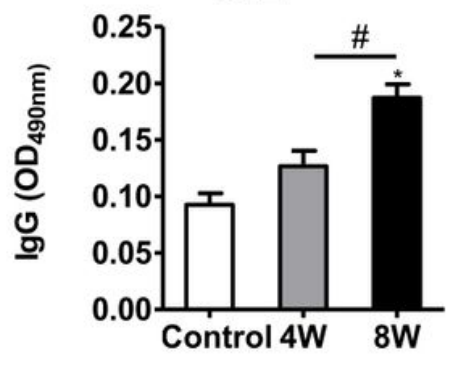

E

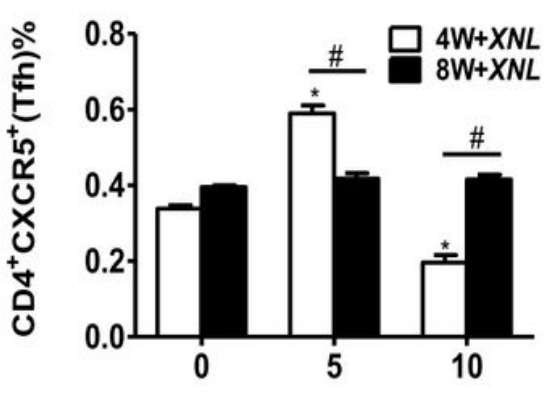

Days post infection(d)

$X N L$

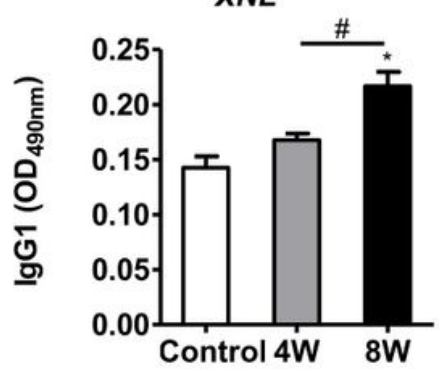

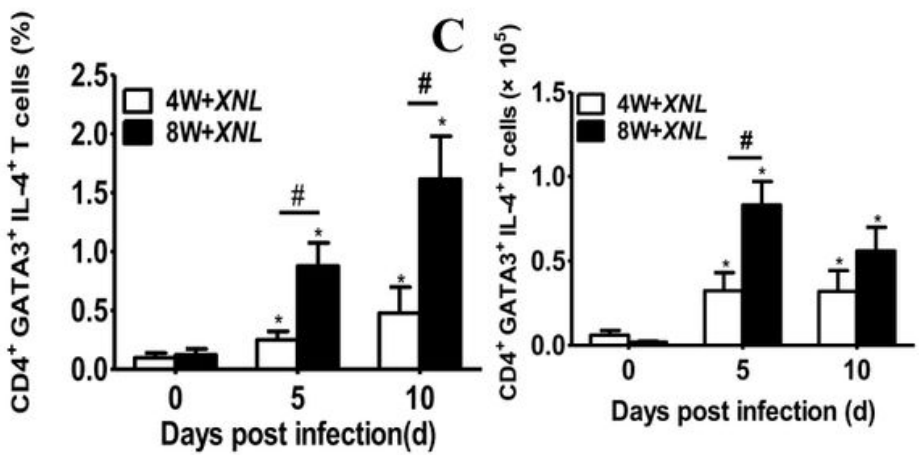

F

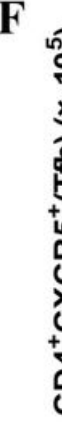

$\mathbf{J}$

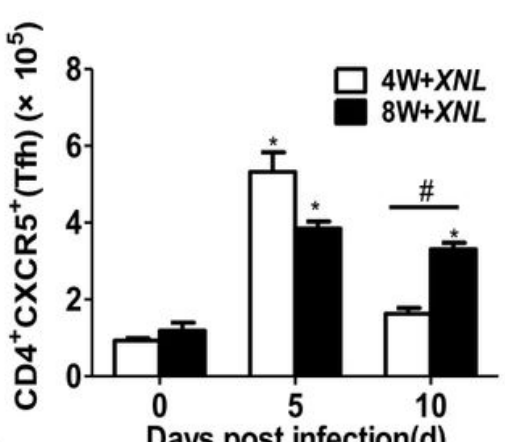

Days post infection(d)

$X N L$

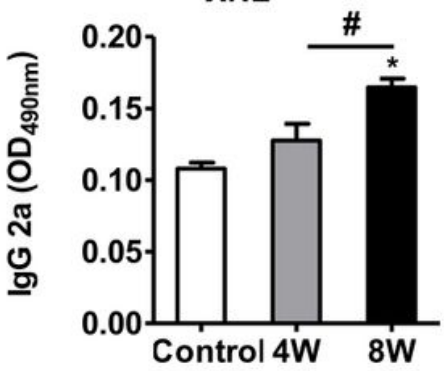

Figure 3

Flow cytometric and ELISA analysis demonstrated Th2 immune response in different species of Plasmodium-infected 4-week-old and 8-week-old BALB/c mice. Representative dot plots (left-upper panel) (A) andcolumn diagram (left-upper panel, the proportion of Th2 cells in CD4+ T cells, absolute cell number) (B) of CD4+GATA3+IL-4+ Th2 cells in 4-week-old and 8-week-old BALB/c mice after Py17XNL infection are displayed. Level of IL-4 (C) in spleen cell supernatants in Py17XNL-infected BALB/C mice were measured. The proportion and absolute cell number of CD4+CXCR5+Tfh cells were measured by flow cytometry in 4-week-old and 8-week-old BALB/c mice after Py17XNL infection (D). Results are presented as arithmetic mean of 3 mice per group \pm SE. Single asterisk $(*)$ and double asterisks $(* \star)$ indicate $p<0.05$ and $p<0.01$, respectively, as compared to control mice. A single pound sign (\#) and a double pound sign (\#\#) indicate $p<0.05$ and $p<0.01$, respectively, as compared to 8-week-old mice. The data are representative of two separate experiments. 
A
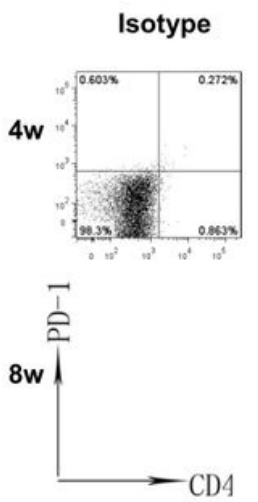

D
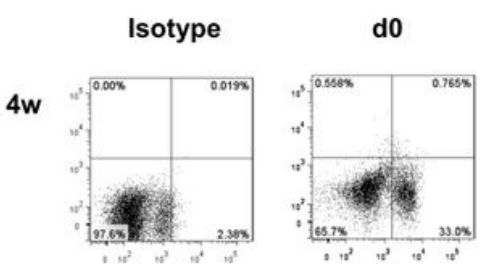

$8 w$

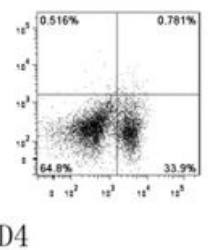

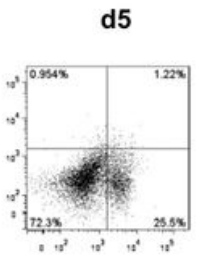

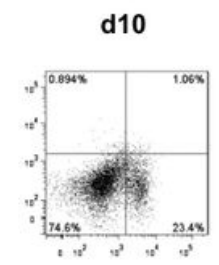

B

d10
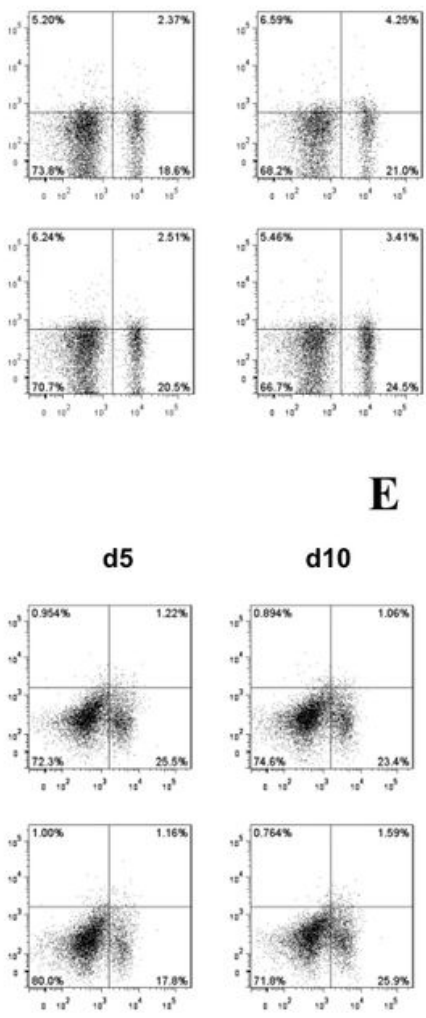

$\mathbf{E}$
C

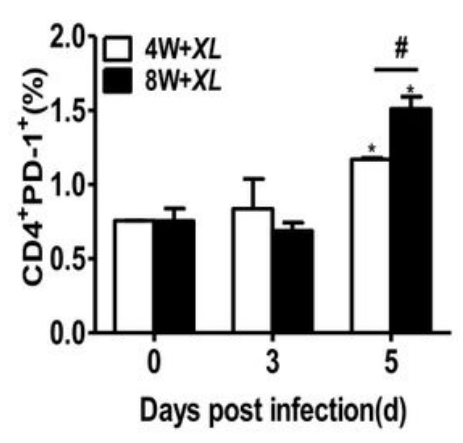

$\mathbf{F}$

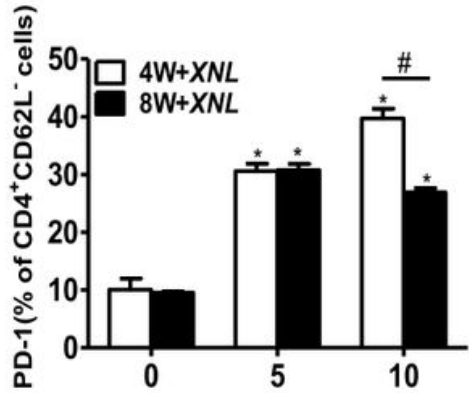

Days post infection(d)
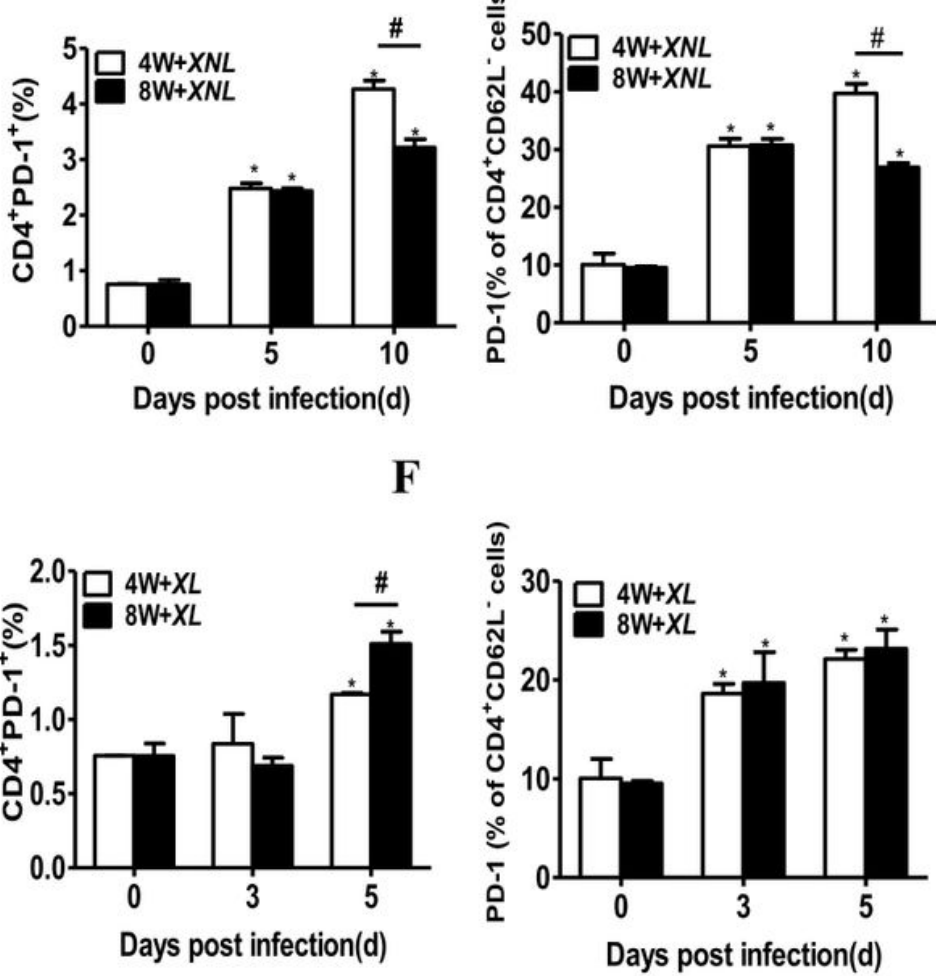

\section{Figure 4}

Flow cytometric analysis demonstrated PD-1 signaling promoted immune response in different species of Plasmodium-infected 4-week-old and 8-week-old BALB/c mice. Representative dot plots (left-upper panel) (A) andcolumn diagram (left-upper panel, the proportion of CD4+PD-1+ cells in splenocytes, absolute cell number) (B) ofCD4+PD-1+ cells in 4-week-old and 8-week-old BALB/c mice after Py17XNL infection are displayed. Representative dot plots (left-lower panel) (C) andcolumn diagram (left-lower panel, the proportion of CD4+PD-1+ cells in splenocytes, absolute cell number) (D) ofCD4+PD-1+ cells in 4-week-old and 8-week-old BALB/c mice after Py17XL infection are displayed. Results are presented as arithmetic mean of 3 mice per group \pm SE. Single asterisk $(*)$ and double asterisks $(* *)$ indicate $p<0.05$ and $p<0.01$, respectively, as compared to control mice. A single pound sign (\#) and a double pound sign (\#\#) indicate $p<0.05$ and $p<0.01$, respectively, as compared to 8-week-old mice. The data are representative of two separate experiments. 
A

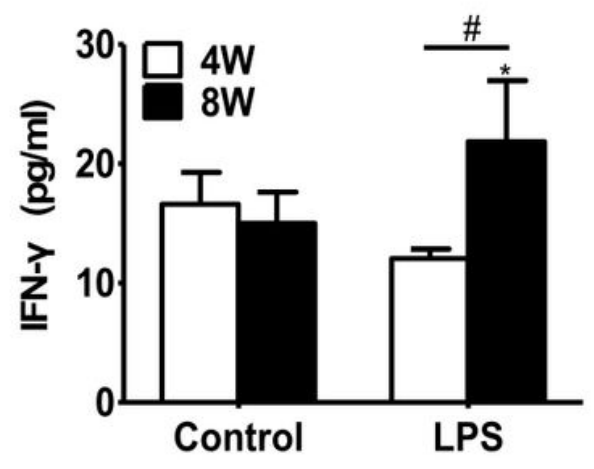

B

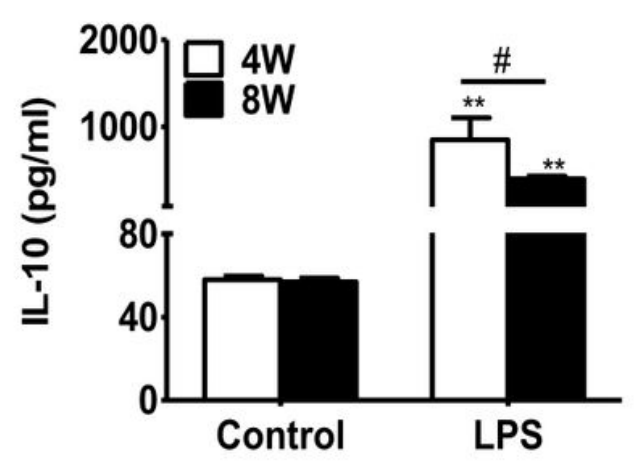

C

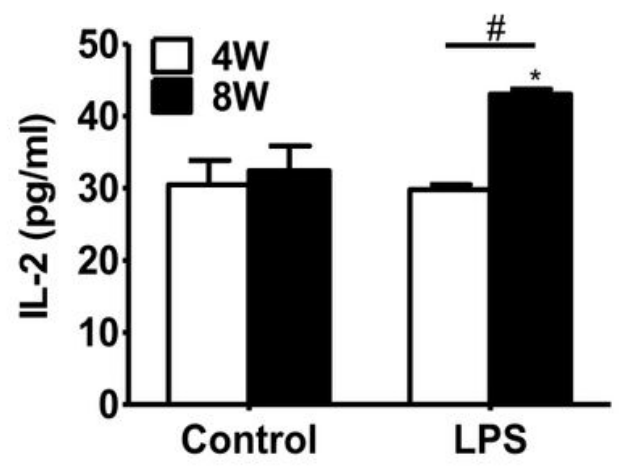

\section{Figure 5}

ELISA analysis demonstrated the levels of total and P. y MSP-1-specific antibodies in spleen supernatants of P. y17XNL-infected 4-week-old and 8-week-old BALB/c mice. IgG (A), IgG1(B), IgG2a (C) and P. y MSP-1specific lgG (D) were measured in supernatants of P. y17XNL-infected 4-week-old and 8-week-old BALB/C mice by ELISA. Results are presented as arithmetic mean of 3 mice per group \pm SE. Single asterisk $\left({ }^{*}\right)$ and double asterisks $\left({ }^{*}\right)$ indicate $p<0.05$ and $p<0.01$, respectively, as compared to control mice. A single pound sign (\#) and a double pound sign (\#\#) indicate $p<0.05$ and $p<0.01$, respectively, as compared to 8-week-old mice. The data are representative of two separate experiments.

\section{Supplementary Files}

This is a list of supplementary files associated with this preprint. Click to download.

- NC3RsARRIVEGuidelinesChecklistfillable.pdf 